\title{
Determinants, outcomes and costs of ceftriaxone v. amoxicillin-clavulanate in the treatment of community- acquired pneumonia at Witbank Hospital
}

\author{
S N Xaba, MB BCh, Dip HIV Man (SA); O Greeff, MB ChB, MPharmMed, MD, FFRP, FCFP; P Becker, BSc, MSc, PhD \\ Department of Pharmacology, Faculty of Health Sciences, University of Pretoria, South Africa
}

Corresponding author: O Greeff(oppel.greeff@up.ac.za)

Background. Community-acquired pneumonia (CAP) is a major cause of death and morbidity worldwide. Treatment is centred on antibiotics with ceftriaxone and amoxicillin-clavulanate being some of the most commonly prescribed agents.

Objective. To compare treatment outcomes and costs in patients receiving either of these two antibiotics at Witbank Hospital (WH).

Methods. A total of 200 randomly selected adult patient files (100 receiving ceftriaxone and 100 amoxicillin-clavulanate) recording a diagnosis of CAP were studied to determine the length of hospital stay, comorbid conditions and treatment outcomes. A descriptive and comparable analysis was performed.

Results. Male gender, higher CURB-65 scores and death were associated with the use of ceftriaxone. Severity of disease and previous antibiotic exposure influenced the duration of hospital admission.

Conclusion. Gender and severity of disease (based on the CURB-65 score) were the determinants of antibiotic choice at WH. Male gender increased the likelihood of being treated with ceftriaxone, as did a CURB-65 score of $>2$. There were no differences in the outcomes of CAP patients treated with ceftriaxone compared with those treated with amoxicillin-clavulanate. Irrespective of antibiotic used, gender and severity of disease influenced treatment outcomes. Male gender was associated with a higher mortality and longer hospital stay. The average duration of stay for both antibiotics was not significantly different. Thus, only level 1 and 2 costs need to be considered when comparing the two regimens. On this basis, ceftriaxone was cheaper than amoxicillin-clavulanate.

S Afr Med J 2014;104(3):187-191. DOI:10.7196/SAMJ.7243

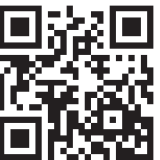

Community-acquired pneumonia (CAP) is the most common type of lower respiratory tract infection and can be life-threatening, especially among young children, the elderly and those with comorbid conditions. ${ }^{[1]}$ The most common pathogen associated with CAP is Streptococcus pneumoniae. ${ }^{[2]}$ Males generally suffer a more severe form of the disease with a higher associated mortality. ${ }^{[3]}$ For those with diabetes mellitus, pneumonia is associated with more severe presentation, poor prognosis and poor outcomes. ${ }^{[4,5]} \mathrm{HIV}$ infection is also a major risk factor for pneumonia.

CAP symptoms are non-specific and patients may present with both upper and lower respiratory tract symptoms. There is no rapid, simple, accurate and cost-effective method for immediate diagnosis of CAP in most patients at presentation. A chest radiograph is necessary to establish the diagnosis of CAP and to differentiate it from other respiratory illnesses. ${ }^{[6]}$ The decision whether or not to admit a patient influences the extent of diagnostic testing as well as the choice of empirical antimicrobial treatment. Pharmacokinetics, efficacy, safety profile, cost, spectrum of activity and whether or not a specific pathogen is identified influence antibiotic treatment choice. Several clinical societies worldwide have authored treatment guidelines for CAP as a way of standardising chemotherapeutic treatment. ${ }^{[7-9]}$

\section{Methods}

Our research was conducted at Witbank Hospital (WH), a 350-bed secondary-level hospital in Mpumalanga Province affiliated to the University of Pretoria as a satellite facility. Clinical records of patients who were treated with ceftriaxone or amoxicillin-clavulanate for CAP, as diagnosed by the treating clinician, from January 2010 to January 2012 were selected and categorised according to the antibiotic selected. For each group, every fourth file was selected beginning with a random number until a total of 100 were reached. This gave a total of 200 randomly selected files with half being treated with amoxicillin-clavulanate and the other half with ceftriaxone. Patients were of any race, gender and over 13 years of age.

To establish the determinants of choice of antibiotic, we examined the severity of disease, presence of comorbid conditions, previous hospital admission/antibiotic exposure, contraindications, ease of administration of either drug and its availability, or lack thereof. To determine treatment outcomes, we established the number of those who: (i) were successfully treated/discharged from hospital; (ii) had complications, which might or might not have been directly related to the choice of antibiotic; and (iii) died. Successful treatment was defined by the resolution of symptoms and by recorded resolution of pneumonic changes on chest radiograph and/or improvement of inflammatory markers (C-reactive protein and white cell count). Death was confirmed by a record in the patient file, as were complications related to the drugs.

Treatment costs were determined by ascertaining the direct costs of acquisition of the antibiotics. Costs related to $(i)$ the diagnosis and treatment of pneumonia; (ii) antibiotic administration; and (iii) hospital accommodation were determined by interviewing relevant hospital personnel. Costs related to other antibiotics used in combination with the study antibiotics were similar as the same antibiotic (erythromycin) was used. 
This study was approved by the Ethics Committee of the University of Pretoria.

\section{Statistical analysis}

A descriptive and comparable analysis was performed. Continuous variables were analysed using the Mann-Whitney $U$ test. Univariate analysis was based on the $\chi^{2}$ test for qualitative variables, while Student's $t$-test was used for quantitative variables. Non-parametric tests were used in the absence of a normal distribution. A logistic model was used to adjust for the effect of multiple variables such as gender, HIV status and severity of disease. A $p$-value of $<0.05$ was considered significant.

\section{Results}

Results are summarised in Table 1. In addition to the comorbidities listed, five patients were receiving amoxicillin-clavulanate, and five were receiving ceftriaxone. One patient receiving ceftriaxone had unexplained hepatitis, with negative viral studies.

Logistic regression was performed to ascertain whether HIV, gender, severity of illness (the CURB-65 score) and previous antibiotic exposure were determinants of antibiotic choice (Table 2). Severity of pneumonia was assessed using the CURB-65 score, which includes fives components: $\mathbf{C}$ - confusion; $\mathbf{U}$ - urea $>7 \mathrm{mmol} / \mathrm{l} ; \mathbf{R}$ - respiratory rate $>30$ breaths/min; $\mathbf{B}$ - blood pressure, systolic $<90 \mathrm{mmHg}$ or diastolic <60 mmHg; and 65 - age $>65$ years. ${ }^{[10]} \mathrm{HIV}$ was not a determinant of antibiotic treatment choice $(p=0.51)$. Male gender was significantly associated with the use of ceftriaxone $(p=0.001)$. A higher CURB-65 score was also significantly associated with the use of ceftriaxone (moderate CURB-65 score: $p=0.003$; severe CURB65 score: $p=0.002$ ). Previous antibiotic exposure was associated with a slightly higher use of ceftriaxone, but this was statistically insignificant $(p=0.799)$.

From multivariate logistic regression, it was established that only gender and CURB-65 score were associated with ceftriaxone treatment (Table 3 ). There was also a significant interaction between gender and CURB-65 score $(p=0.046)$.

For death as an outcome, choice of antibiotic, HIV status, gender, severity of disease and previous antibiotic exposure were analysed by logistic regression. Ceftriaxone showed a significant association with death as an outcome $(p=0.032)$. HIV showed no significant influence $(p=0.459)$. There was a higher incidence of deaths among males. A significant association between the severity of disease and death was observed $(p=0.029)$. Previous antibiotic exposure was not associated with death $(p=0.312)$.

Treatment with ceftriaxone was associated with a slightly longer length of hospital stay than amoxicillin-clavulanate; however, the difference was not statistically significant (mean 6.21 v. 5.22 days, respectively; $p=0.093$ ). The HIV-positive status of patients led to a slightly longer hospital stay after treatment with ceftriaxone v. amoxicillin-clavulanate; however, this was not statistically significant (mean 6.04 v. 4.49 days, respectively; rank-sum $p=0.253$ ). There was no association between gender and length of stay (rank-sum $p=0.477)$. Severity of disease did have an influence on the length of stay, with moderate disease leading to an average of 5.04 days in hospital and severe disease leading to an average of 7.01 days in hospital (rank-sum $p=0.015$ ). Previous antibiotic exposure also led to a slightly longer hospital stay than no previous exposure (mean 6.97 v. 5.12 days, respectively; rank-sum $p=0.015$ ), which is statistically significant.

From multivariate logistic regression analysis, death was associated with ceftriaxone, a high CURB-65 score and male gender. When
Table 1. Demographic data

\begin{tabular}{cll}
\hline & Ceftriaxone & $\begin{array}{l}\text { Amoxicillin- } \\
\text { clavulanate }\end{array}$ \\
\hline $\begin{array}{l}\text { Patients, } N \\
\text { Age (years) }\end{array}$ & 100 & 100 \\
Range & $15-83$ & $14-89$ \\
Average & 45.73 & 41.37 \\
Median & 49 & 37.5 \\
Gender, $n$ & & \\
Male & 89 & 46 \\
Female & 11 & 54
\end{tabular}

Length of hospital

stay (days)

$\begin{array}{lll}\text { Average } & 6.22 & 5.18 \\ \text { Median } & 5 & 5\end{array}$

Patients with previous $\quad 34 \quad 32$

antibiotic exposure, $n$

CURB-65 score, average $\quad 2.4 \quad 2.1$

Comorbidities, $n$

HIV $43 \quad 39$

$\begin{array}{lll}\text { T2DM } & 5 & 2\end{array}$

Epilepsy $\quad 1 \quad 2$

COPD 3

Heart failure $\quad 2 \quad 6$

Ischaemic stroke $\quad 2$

Pneumonia-related

complications, $n$

$\begin{array}{lll}\text { Pleural effusion } & 1 & 5\end{array}$

Septic shock $\quad 0 \quad 1$

Deep vein thrombosis 11

Lung abscess $\quad 2 \quad 0$

Outcomes, $n$

$\begin{array}{lll}\text { Complications } & 6 & 7\end{array}$

Patients successfully $\quad 73 \quad 88$

treated (discharged)

Deaths

27

12

T2DM = type 2 diabetes mellitus; $\mathrm{COPD}=$ chronic obstructive pulmonary disease.

controlled for gender, treatment was not significantly associated with outcome $(p=0.191)$ while severity of disease was associated with outcome $(p=0.05)$.

\section{Discussion}

\section{Determinants and treatment outcomes}

When comparing determinants and outcomes of CAP in the two antibiotic groups we found that gender played an important role in antibiotic choice, with $>80 \%$ of males prescribed ceftriaxone. Additionally, more males in our study had a more severe form of pneumonia, as exemplified by a higher CURB-65 score. This is in line with previous studies; male gender is a risk factor for developing severe pneumonia with higher mortality. 
Severity of disease also played an important role in the choice of antibiotic. Patients with higher CURB-65 scores were more likely to receive ceftriaxone than amoxicillinclavulanate. That ceftriaxone was prescribed for sicker patients may suggest that doctors believe that ceftriaxone is superior to amoxicillin-clavulanate. Studies have shown the cure rates of ceftriaxone to be $84 \%$ and that of amoxicillin-clavulanate to be $76 \%,{ }^{[1,12]}$ a fact that might have influenced antibiotic choice in CAP treatment by clinicians at $\mathrm{WH}$.

Severity of disease was also associated with a longer hospital stay, the link between severity of disease and hospital length of stay is well documented. ${ }^{[13]}$ However, as Fine et al. ${ }^{[14]}$ revealed, and as was subsequently confirmed by others, ${ }^{[15]}$ the length of hospital stay for treatment of CAP is not solely determined by patient-dependent factors such as age, severity of disease and the presence of comorbid conditions, but is rather heterogeneous with great variation between hospitals reflecting physician- and hospital-specific behaviours. There is no reason to believe that this was the case in the current study, given the economic and capacity challenges under which WH operates.

HIV-positive status proved not to be a determinant of antibiotic choice. Notably, $41 \%$ of patients in our study were HIVpositive, but we found no link between their status and choice of antibiotic regimen.

Prior antibiotic exposure is thought to be a major risk factor for antibiotic resistance. ${ }^{[16]}$ However, in our study, it was not a determinant. This is not surprising because based on analysis of published literature on CAP resistance, Feldman ${ }^{[17]}$ concluded that 'little evidence suggests that infections with resistant pneumococci per se are more severe.'

In comparing treatment outcomes, we looked at death or cure and also compared different variables looking at the length of hospital stay. We found that more patients receiving ceftriaxone died compared with those receiving amoxicillin-clavulanate. This is because sicker patients (most of whom were male) were prescribed ceftriaxone. However, in the multivariate analysis used to predict mortality, gender and severity of disease were found to be associated independently with a higher mortality

\begin{tabular}{|c|c|c|c|c|}
\hline Determinant & $\%$ ( $n / N$ cases $)$ & Crude OR & $95 \% \mathrm{CI}$ & $p$-value \\
\hline \multicolumn{5}{|l|}{ HIV } \\
\hline Positive & $52.4(43 / 82)$ & 1.21 & $0.688-2.125$ & 0.510 \\
\hline Negative & $48.3(57 / 118)$ & 1.00 & & \\
\hline \multicolumn{5}{|l|}{ Gender } \\
\hline Female & $16.9(11 / 65)$ & 1.00 & $3.947-16.679$ & 0.001 \\
\hline Male & $65.9(89 / 135)$ & 8.11 & & \\
\hline \multicolumn{5}{|l|}{ CURB-65 Score } \\
\hline Mild $(0-1)$ & $5.0(1 / 20)$ & 1.00 & & \\
\hline Moderate (2) & $54.0(60 / 112)$ & 22.35 & $2.891-172.81$ & 0.003 \\
\hline Severe $(3-4)$ & $57.4(39 / 68)$ & 25.55 & $3.232-201.98$ & 0.002 \\
\hline \multicolumn{5}{|c|}{$\begin{array}{l}\text { Previous antibiotic } \\
\text { exposure }\end{array}$} \\
\hline Yes & $51.6(33 / 64)$ & 1.08 & $0.596-1.959$ & 0.799 \\
\hline No & $49.6(67 / 135)$ & 1.00 & & \\
\hline
\end{tabular}

and, after adjusting for their confounding effect, there were no statistically significant differences between the two antibiotic regimens. The ceftriaxone group had a longer duration of stay, averaging 6.22 days compared with 5.18 days in the amoxicillinclavulanate group. This was not statistically significant and the length of hospital stay may have been related to factors such a severity of disease and presence of comorbid conditions, rather than choice of antibiotic.

In our study, HIV did not increase the risk of CAP-related death. The influence of HIV on CAP treatment outcomes has been the subject of several studies. In three of these studies, using univariate analyses of clinical outcomes of CAP, higher death rates were found among the HIV-infected patients. ${ }^{[1-20]}$ However, not surprisingly, the higher death rates were among patients who were not receiving highly active antiretroviral therapy (HAART). Bordon et al. ${ }^{[2]}$ conducted a study to evaluate the clinical outcomes of CAP among hospitalised HIV-infected patients and found that HIV did not significantly influence overall mortality rates or CAPrelated mortality rates. Even though a higher number of HIV-positive patients with pneumonia died in our study (20\% HIVpositive v. $16 \%$ HIV-negative, irrespective of antibiotic choice), this was not statistically significant in line with Bordon et al's findings.

With respect to length of hospital stay, the influence of HIV was also statistically insignificant. Johnson et al. ${ }^{[22]}$ reported a longer hospital stay among HIV-infected patients. However, the patients studied were not receiving HAART. Studies have confirmed the link between introducing HAART and a reduction in the incidence of bacterial pneumonia cases. ${ }^{[23]}$

Gender influence on death and length of stay as outcomes was statistically insignificant. Thus, while males are known to be more likely to suffer from a severe form of CAP, our results, based on a small sample size, suggest that they do not necessarily stay longer in hospital and that they are not more likely to die. This calls for a much larger sample size in future studies as these results are at odds with previous studies. ${ }^{[3,24]}$

Table 3. Summary of results

\begin{tabular}{lllll}
\hline Variables & Determinant of choice of antibiotic & Outcome & Length of hospital stay & Treatment cost \\
\hline HIV & Nil association & Nil association & $\begin{array}{l}\text { Longer stay but not } \\
\text { significant }\end{array}$ & Nil association \\
Gender & Significant difference & Males more affected & Nil association & Nil association \\
CURB-65 score & Significant difference & Significant difference & Significant difference & Significant difference \\
Previous antibiotic exposure & Association but not significant & Nil association & Significant difference & Significant difference
\end{tabular}


Severity of disease (higher CURB-65 score) had a statistically significant influence on death as a treatment outcome irrespective of antibiotic regimen. The CURB-65 score also had a statistically significant influence on the length of hospital stay. These results are in line with those from a recent study by Armiñanzas et al. ${ }^{[25]}$ However, the mean age in Armiñanzas et al's study of 539 patients ( $51 \%$ male) was 78 years, which is much higher than in our study.

The association between death as an outcome and previous antibiotic exposure, which could suggest potential resistance, was statistically insignificant. There was also no statistically significant relationship between the length of hospital stay and previous antibiotic exposure. Interestingly, from our results, the group that had been previously exposed to antibiotics had fewer deaths although a longer hospital stay, giving the impression that previous exposure to antibiotics ( 3 months prior to admission) in CAP is protective and reduces the risk of death. For amoxicillin-clavulanate at least, this is in agreement with the results from the case-control study by Einarsson et al. ${ }^{[26]}$

Of interest, in our study, we observed that the mean age difference (Table 2) was statistically insignificant. Therefore, age as a factor did not independently contribute to the choice of antibiotic, or to any of the outcomes.

\section{Complications and comorbid conditions}

In addition to the comorbidities listed in Table 1, there was one case of prostate cancer, two of underlying fibrotic lung disease, three of epilepsy, seven of diabetes, three of stroke and four of chronic obstructive pulmonary disease. None of the seven diabetic patients in our study (two receiving amoxicillin-clavulanate and five receiving ceftriaxone) died and none stayed longer than the median 5 days. This is in contrast with a study by Moosa et al ${ }^{[27]}$ which showed an increased risk of mortality and morbidity. Although statistically insignificant, with respect to death as an outcome, the results in diabetes patients are in line with finding by Belk et al. ${ }^{[28]}$ that, after controlling for other variables, patients with type 2 diabetes mellitus had a lower risk of in-hospital mortality (but longer length of stay) when hospitalised for pneumonia.

\section{Costs implications}

Pharmaco-economic analysis can be classified into three levels of costs: ${ }^{[29]}$ (i) level 1 (pharmacy perspective) considers only the acquisition price of the study medication; (ii) level 2 adds to the level 1 costs all other costs directly related to antibiotic use and infection treatment - antibiotic-related items include medication preparation, dispensing and administration, therapeutic drug monitoring, treatment of adverse events and secondary treatment for failures; and (iii) level 3 costs include all level 2 items and other hospital costs incurred during treatment. The daily cost of occupying a hospital bed is highly variable and depends on the type of unit (intensive care or general), the levels of technology and services provided, and geographic location.

Because the length of hospital stay in both groups showed no statistically significant differences, level 3 costs were similar for both treatment regimens. With level 2 costs, all other contributors were rendered irrelevant by similar treatment outcomes between the two antibiotics. In addition, there were no complications related to antibiotic use for both regimens. The only relevant aspect under level 2 costs was the frequency of administration of the antibiotic, with amoxicillin-clavulanate, because of its shorter half-life, requiring three injections in a 24-hour period while ceftriaxone requires oncea-day administration. This makes ceftriaxone more cost-effective.
With respect to level 1 costs, ceftriaxone costs R3.40 per vial and requires $3.5 \mathrm{ml}$ of sterile water to reconstitute and is given as a daily parenteral dose. On the other hand, amoxicillin-clavulanate costs R11.80 per vial, requires $20 \mathrm{ml}$ of sterile water for reconstitution and is given three times a day. The oral formulations cost R11.88 for 875 $+175 \mathrm{mg}$ (10s) and R11.30 for $250+125 \mathrm{mg}$ (15s). Thus, ceftriaxone is $>70 \%$ cheaper than amoxicillin-clavulanate over an average treatment period of 5 days. The above antibiotic prices are based on the latest tender prices at the time of this study.

\section{Conclusion}

Gender and severity of disease (CURB-65 score) were determinants of antibiotic choice in WH. Male gender predisposed patients to a ceftriaxone regimen as does a CURB- 65 score of $>2$. There were no differences in the outcomes of CAP patients treated with ceftriaxone and those treated with amoxicillin-clavulanate. Irrespective of the antibiotic used, gender and severity of disease had an influence on treatment outcomes. Male gender was associated with a higher mortality and longer hospital stay; however, the average length of stay for both antibiotics was not significantly different.

HIV status was neither a determinant of antibiotic choice nor had any influence on treatment outcomes. Our study demonstrated that there were no differences between the two groups of antibiotics and that severity of disease and gender have a large influence on the choice of antibiotic. When CAP patients were stratified by HIV status, no significant differences existed between the HIV-negative and HIV-positive groups. Since length of hospital stay was not different in either case, only level 1 and 2 costs needed to be considered when comparing the two regimens. On this basis, ceftriaxone is cheaper than amoxicillin-clavulanate.

While previous exposure to antibiotics was noted, the specific antibiotic was not noted from each patient. Another limitation of the study is that even though the HIV status of the subjects was noted, whether these patients were receiving HAART or not, the severity of the immune-suppression ( $\mathrm{CD} 4^{+}$count) and for those receiving HAART, the specific combination of drugs they were receiving, were not taken into consideration.

For its random nature with respect to patient file selection, there were more males in this study than females. All patients in the study were recruited from the general medical wards. Cigarette smoking, a significant risk factor in the development of pneumonia was not studied. Nonetheless, this study provided data on which more research can be based to expand the economic impact of treatment choice in the management of CAP.

Acknowledgements. The authors thank WH management and staff for their cooperation. Expert advice from Prof. Paul Rheeder is also acknowledged.

\section{References}

1. Marrie TJ. Community-acquired pneumonia. Clin Infect Dis 1994;18(4):501-513.

2. File TM Jr. Community-acquired pneumonia. Lancet 2003;362(9400):1991-2001. [http://dx.doi, org/10.1016/S0140-6736(03)15021-0]

. Falagas EM, Mourtzoukou EG, Vardakas KZ. Sex differences in the incidence and severity of respiratory tract infections. Respir Med 2007;101(9):1845-1863. [http://dx.doi.org/10.1016/j.rmed.2007.04.011] 4. Moosa PE, Phrabhakar K, Lakshmaiah V, Jayarama N. Study of bacterial pneumonia in type 2 diabetes clinical profile and outcome. Int J Infect Dis 2011;15(Suppl 1):S52. [http://dx.doi.org/10.1016/S12019712(11)60178-1]

5. Klein OL, Smith LJ, Tipping M, Peng J, Williams MV. Reduced diffusion lung capacity in patients with type 2 diabetes mellitus predicts hospitalization for pneumonia. Diabetes Res Clin Pract Suppl 2011;92(1): el2-e15. [http://dx.doi.org/10.1016/j.diabres.2010.12.012]

6. Metlay JP, Kapoor WN, Fine MJ. Does this patient have community acquired pneumonia? JAMA 1997;278(17):1440-1445. [http://dx.doi.org/10.1001/jama.278.17.1440]

7. Niederman MS, Luna CM. Community-acquired pneumonia guidelines: A global perspective. Semin Respir Crit Care Med 2012;33(3):298-310. [http://dx.doi.org/10.1055/s-0032-1315642]

8. The British Thoracic Society; Community Acquired Pneumonia in Adults Guideline Group. Guidelines for the management of community acquired pneumonia in adults: Update 2009. Thorax 2009;64(Suppl III):iiil-iii55. [http://dx.doi.org/10.1136/thx.2009.121434] 
9. Feldman C, Brink A, Bateman GA, Maartens G, Bateman ED. Management of community-acquired pneumonia in adults. S Afr Med J 2007;97(12):1296-1306.

10. Lim WS, Van der Eerden MM, Laing R, et al. Defining community acquired pneumonia severity on presentation to hospital: An international derivation and validation study. Thorax 2003:58(5):377-382. [http://dx.doi.org/10.1136/thorax.58.5.377]

11. De Klerk GJ, Van Steijn JHM, Lobatto S, et al. A randomised, multicentre study of ceftriaxone versus standard therapy in the treatment of lower respiratory tract infections. Int I Antimicrob Agents 1999;12(2):121-27. [http://dx.doi.org/10.1016/S0924-8579(99)00037-0]

12. Ball P. The clinical development and launch of amoxicillin/clavulanate for the treatment of a range of community-acquired infections. Int J Antimicrob Agents 2007;30(Suppl 2):113-117. [http://dx.doi. org/10.1016/j.ijantimicag.2007.07.037]

13. Capelastegui A, España PP, Quintana JM, et al. Management of community-acquired pneumonia and secular trends at different hospitals. Respir Med 2005;99(3):268-278. [http://dx.doi.org/10.1016/j. rmed.2004.08.010]

14. Fine MJ, Singer DE, Phelps AL, Hanusa BH, Kapoor WN. Differences in length of stay in patients with community-acquired pneumonia: A prospective four-hospital study. Med Care 1993;31(4):371-380. [http://dx.doi.org/10.1097/00005650-199304000-00008]

15. Feagan BG, Marrie TJ, Lau CY, et al. Treatment outcomes of community-acquired pneumonia at Canadian hospitals. Can Med Assoc J 2000;162(10):1415-1420.

16. Bauer T, Ewig S, Marcos MA, Schultze-Werninghaus G, Torres A. Streptococcus pneumoniae in community-acquired pneumonia. How important is drug resistance? Med Clin North Am 2001;85:1367-1379.

17. Feldman C. Clinical relevance of antimicrobial resistance in the management of pneumococcal community-acquired pneumonia. J Lab Clin Med 2004;143(5):269-283. [http://dx.doi.org/10.1016/j. lab.2004.02.002]

18. Feldman C, Klugman KP, Yu VL, et al. Bacteraemic pneumococcal pneumonia: Impact of HIV on clinical presentation and outcome. J Infect 2007;55(2):125-135. [http://dx.doi.org/10.1016/j. jinf.2007.04.001

19. Park DR, Sherbin VL, Goodman MS, et al. Harborview CAP Study Group. The etiology of community acquired pneumonia at an urban public hospital: Influence of HIV infection and initial severity of illness. J Infect Dis 2001;184(3):268-277. [http://dx.doi.org/10.1086/322040]
20. Touchie C, Marrie TJ. Comparison of community-acquired pneumonia requiring admission to hospital in HIV- and non-HIV-infected patients. Can J Infect Dis 1996;7(4):253-258.

21. Bordon J, Kapoor R, Martinez C, et al. $\mathrm{CD}^{+}$cell counts HIV-RNA levels do not predict outcomes of community acquired pneumonia in hospitalized HIV infected patients. Int $\mathrm{J}$ Infect Dis 2011:15(12):e822-e827. [http://dx.doi.org/10.1016/jijid.2011.05.021]

22. Johnson DH, Carriere KC, Houston S. Hospitalization for community-acquired pneumonia in Alberta patients with human immunodeficiency virus infection: A case control study. Can Resp J 2003;10(5):265-270.

23. De Gaetano Donati K, Bertagnolio S, Tumbarelo M, et al. Effect of highly active antiretroviral therapy on the incidence of bacterial pneumonia in HIV infected subjects. Int J Antimicrob Agents 2000;16(3):357-36 24. Gordon HS, Rosenthal GE. The relationship of gender and in-hospital death: Increased risk of death in men Med Care 1999;37(3):318-324. [http://dx.doi.org/10.1097/00005650-199903000-00011]

25. Armiñanzas $\mathrm{C}$, Velasco L, Calvo, $\mathrm{N}$, et al. CURB-65 as an initial prognostic score in internal medicine patients. Eur J Intern Med 2013;24(5):416-419. [http://dx.doi.org/10.1016/.ejim.2013.01.004]

26. Einarsson S, Kristjansson M, Kristinsson KG, Kjartansson G, Jonsson S. Pneumonia caused by penicillinnon-susceptible and penicillin-susceptible pneumococci in adults: A case-control study. Scand J Infect Dis 1998;30(3):253-256.

27. Moosa PE, Prabhakar K, Lakshmaiah V, Jayarama N. PP-025 Study of bacterial pneumonia in type 2 diabetes - clinical profile and outcome. Int J Infect Dis 2011;15(Suppl 1):S52. [http://dx.doi.org/10.1016/ S1201-9712(11)60178-1]

28. Belk KW, Craver CW, Blanchette CM. PDB25 Impact of diabetes in patients hospitalized for pneumonia Value in Health 2012;15(4):A175. [http://dx.doi.org/10.1016/j.jval.2012.03.946]

29. Dresser LD, Niederman MS, Paladin JA. Cost-effectiveness of gatifloxacin vs ceftriaxone with a macrolide for the treatment of community-acquired pneumonia. Chest 2001;119(5):1439-1448. [http://dx.doi org/10.1378/chest.119.5.1439]

Accepted 16 October 2013. 